\title{
Impact of Fixed Choice Design on Blockmodeling Outcomes
}

\author{
Anja Žnidaršič ${ }^{1}$
}

\begin{abstract}
The main goal of blockmodeling is to reveal the essential structure of the network based on patterns of relationships. Social network data usually contain different types of errors and one of them is caused by some limitation of number of selected actors in the research design. The impact of fixed choice design compared to free choice design on the results of blockmodeling are studied through simulations. The resulting blockmodels are compared with two indices where position membership of actors and the structure of the blockmodels are examined. Limiting the number of actors that can be selected has an impact on delineated blockmodel structure where the deletion of ties has higher effect than addition of them.
\end{abstract}

\section{Introduction}

Social networks which are gathered usually with surveys and questionnaires (Marsden, 2005, 2011; Wasserman and Faust, 1994) are measured with errors. According to the definition by Holland and Leinhardt (1973) an error in a social network occurs when there is an extra tie or a missing tie according to the true underlying and unobservable structure. Types of errors found in the literature could be classified into three categories (Žnidaršič et al., 2012a): (i) boundary specification problem; (ii) errors caused by questionnaire format; and (iii) errors caused by actors.

The boundary specification problem concerns rules of inclusion for actors in a studied network (Laumann et al., 1983; Doreian and Woodard, 1994; Kossinets, 2006).

Network instruments are another source for introducing errors. Three different question formats are often considered when designing instruments for collecting social network data: (i) free or fixed choice designs (discussed in detail in Section 2); (ii) using recall or recognition of actors (Hlebec, 1993; Brewer, 2000; Hlebec and Ferligoj, 2001; Bell et al., 2007); and (iii) seeking data for directed (e.g., asking about providing or receiving of social support) or symmetric ties (Stork and Richards, 1992; Ferligoj and Hlebec, 1999).

Errors caused by actors can be divided to: (i) (complete) actor non-response (Stork and Richards, 1992; Costenbader and Valente, 2003; Kossinets, 2006; Knoke and Yang, 2008; Huisman, 2009; Žnidaršič et al., 2012a); (ii) tie non-response or absent ties (Rumsey,

\footnotetext{
${ }^{1}$ Faculty of Organizational Sciences, University of Maribor, Kidričeva 55a, 4000 Kranj, Slovenia; anja.znidarsic@fov.uni-mb.si
} 
1993; Borgatti et al., 2006; Huisman and Steglich, 2008; Huisman, 2009; Žnidaršič et al., 2012b); and (iii) measurement errors (Holland and Leinhardt, 1973; Feld and Carter, 2002).

Impacts of actor non-response and item non-response on the stability of blockmodeling were presented by Žnidaršič et al. (2012a,b). Our concern here is when a questionnaire format demands a fixed number of nomination of actors and implications of this for blockmodeling, and, therefore, the study of network structure. The paper is organized as follows: Section 2 considers briefly errors due to fixed and free choice design questionnaire formats and in Section 3 blockmodeling of binary networks is discussed. Section 4 describes the simulations on networks used here. Section 5 presents the results of simulations according of the stability of blockmodels. We finish with conclusions (Section 6) together with some recommendations for researchers.

\section{Fixed or free choice design}

The questionnaire or name generator in the social network collection process can have instructions about predetermined number of actors (or choices) which each network member can select. This question format is known as a fixed choice design while the alternative is a free choice design.

The potential problems of fixed choice designs were pointed out by Holland and Leinhardt (1973). When a fixed number of alters is specified in an measurement instrument, actors with more ties than the threshold are forced to leave out alters and actors with fewer ties can add nonexistent ties to reach the threshold. While it is possible that the true structure is exactly the same as the observed structure with a fixed choice design, it is likely that either the true structure is contained within the observed structure or, even more likely, the observed structure is contained within the real structure ${ }^{2}$. This implies the presence of missing data for specific ties.

"Fixed choice nominations can easily lead to a non-random missing data pattern" (Kossinets, 2006). Popular individuals with many contacts are more likely to be chosen by their friends and friends of your friends are very likely to be your friends too - transitivity patterns in a network (Feld, 1991; Newman, 2003). Marsden (2011: 373) emphasized the practical advantages of fixed choice nominations in survey administration where "they simplify and specify a sociometric task for respondents, thereby reducing burden". However, Newman (2010) emphasized that limitation of number of alters is undesirable for most purposes because it clearly underestimates or limits the outdegree of the actors in the network due to artificial and unrealistic imposed cut-offs.

A free choice design has the potential to allow the collection of richer network data but it does not automatically eliminate errors. These can arise also from respondents having different interpretations of the terms in a question ${ }^{3}$ (Holland and Leinhardt, 1973; Hlebec and Kogovšek, 2006). Additionally, the graphical appearance of the name generator in a web survey can affect responses more than the wording of questions or specific instructions provided by researchers (Lozar Manfreda et al., 2004; Vehovar et al., 2008). These

\footnotetext{
${ }^{2}$ Of course, both problems can be present in the collected network data.

${ }^{3}$ For example, the term 'friend' can have different components ranging from 'acquaintance' to 'best friend'.
} 
problems can also lead to missing specific ties and to some respondents reporting no ties for all other members of the network.

Kogovšek and Hlebec (2005) investigated the effect of limiting the number of actors in egocentric networks. They found only minor differences in network structure and composition comparing data gathered without limitation of number of actors and with a limitation to the first five alters. The main reason for this result was the small size of networks because the majority of them had fewer than six alters and therefore the limitation used does not play a major role. Kogovšek et al. (2010) emphasized that in most studies where some limitation is used, it is in range from three to eight alters, while they restricted the reported number to three alters. They reported that instrumental support is relatively insensitive to using a direct limitation of alters ${ }^{4}$, while higher differences are observed with emotional support where statistically significant differences are observed mainly for strong ties (e.g., percentages of partner, mother, friend). Similar to Newman (2010) for whole networks, Kogovšek et al. (2010:104) suggested that "using a direct limitation seems not to be a universally advisable option and we would advise using it only after careful consideration of the aims of a study".

\section{Blockmodeling}

The purpose of blockmodeling procedures is to partition the network actors into clusters (called positions), and, at the same time, partition the set of ties into blocks which are determined by the ties between actors in pairs of positions (e.g., Wasserman and Faust 1994; Doreian et al., 2005). The actors within a cluster and to the actors of the other clusters should have the same or similar connection patterns based on a selected equivalence. The resulting blockmodel is a smaller representation of a network which reveals its essential structure. The vertices in the reduced graph represent positions of equivalent actors and the arcs (summarizing blocks) represent ties between positions (Doreian et al., 2005).

As noted above, blockmodel partitioning is based on some type of equivalence. The most commonly used type (used also in this paper) is structural equivalence. Actors are structurally equivalent if they are connected to the rest of the network in identical ways (Lorrain and White, 1971). The definition can be written in mathematical notation as follows (Batagelj et al., 1992b; Doreian et al., 2005):

Actors $x_{i}$ and $x_{j}$ are structurally equivalent if and only if

(i) $r_{i j}=r_{j i}$,

(ii) $r_{i i}=r_{j j}$,

(iii) $\forall k \neq i, j: r_{i k}=r_{j k}$,

(iv) $\forall k \neq i, j: r_{k i}=r_{k j}$, where $r_{i j}$ indicates a relation between actors $x_{i}$ and $x_{j}$.

\footnotetext{
${ }^{4}$ The limitation of actors in a survey is a direct one if the limitation of actors is included in the question itself (e.g., please name five people with whom you discuss important matters). The limitation of number of actors is indirect if the respondents are not aware of the limitation because it is not explicitly written in the question. An indirect limitation can arise if detailed data are gathered only for the first few alters later in the survey or if a researchers limit their analysis to the first few alters.
} 
Batagelj et al. (1992b) proved that for structural equivalence there are only two possible ideal blocks: null (zero covered - there are no ties between actors of two clusters) and complete (one covered - each actor from the first cluster is connected to all actors from the second cluster). A generalization of structural equivalence is regular equivalence (Batagelj et al., 1992a) and the concept of generalized equivalence (Doreian et al., 2005 ) is defined by (extending) the set of allowed block types.

Batagelj et al. (1992a, b) distinguished indirect and direct approaches to blockmodeling. In the direct approach a set of permitted block types is fitted to a network which is done by minimizing a compatible criterion function defined for the specific equivalence. Both direct and indirect approaches have been implemented in Pajek (Batagelj and Mrvar, 2012; Mrvar and Batagelj 2012) and in the R-package called Blockmodeling (Žiberna, 2008) which were used in our simulations.

\section{The design of simulation study and data used}

In order to study the impact of the limitation of the number of actors on the obtained blockmodel, simulations based on two real networks were performed. In Section 4.1 the design of simulations is presented; Section 4.2 describes two indices for blockmodel comparisons; and Section 4.3 presents two real whole networks collected with free choice design.

Sometimes the instructions in the fixed choice design questionnaire format allow the respondents to report up to a selected number of choices (e.g., "nominate up to three of your friends"). In this paper the term 'fixed choice' is used for an exact number of desired nominations.

In the simulations the ties were randomly added or randomly deleted (or both) to satisfy the selected criterion of the number of fixed choices (nFixed). If, in the real starting network, actors nominate three to five friends and the limitation criteria of fixed choices is set to only two nominations ties were randomly deleted from the network. If the number of desired fixed choices is restricted to six actors then ties were randomly added to the starting network. The ties were both randomly added and randomly deleted if the number of fixed choices were restricted to four choices. More precisely in the case of having $n$ Fixed $=4$, ties were randomly added to those actors who nominated only three friends and randomly deleted from those actors who nominate five or more friends in the real starting network.

\subsection{A scheme of simulations}

The basic simulation scheme has four main steps:

(i) To establish the blockmodel based on structural equivalence ${ }^{5}$ of a whole (real) network collected with a free choice design having two parts: the (real) partition of actors and the image matrix with the distribution of block types by location.

\footnotetext{
${ }^{5}$ In the simulations only structural equivalence was used because it is the most commonly used type of equivalence and the second reason is that regular and generalized types of equivalence can be extremely unstable if small proportion of errors is introduced to the network data (Žnidaršič, 2012; Žnidaršič et al., 2012c).
} 
(ii) To construct the 'simulated' networks with fixed number of choices, made by selecting some numbers of limitations of choices (denoted as nFixed) on the (real) network. The ties were randomly added or deleted (or both) on the (real) network to meet the limitation criteria ${ }^{6}$.

(iii) To establish a blockmodel based on structural equivalence of the 'simulated' network that results in the 'simulated' partition and the 'simulated' image matrix.

(iv) Comparison of the resulting blockmodels of the real and the 'simulated' network using: (i) The Adjusted Rand Index to compares the two sets of positions; and (ii) the proportion of incorrect blocks (See Section 4.2).

\subsection{Comparison of two blockmodels}

The result of a blockmodeling procedure is a partition of actors that determines positions and the image matrix with selected block types. Therefore, a pair of blockmodels is compared in two different ways.

The first index is the Adjusted Rand Index - ARI (Hubert and Arabie, 1985) which measures the differences between two partitions or, more precisely, measures the concordance between them. Its computation is based on how pairs of actors are placed within two partitions. Lower values of the index signal worse correspondences of the position memberships. It has an expected value 0 (when comparing two random partitions) and its maximal value is 1 . Based on simulations, Steinley (2004) presented some general guidelines for interpreting the Adjusted Rand Index ( $A R I$ ): (i) $A R I \geq 0.9$ indicates excellent agreement between two partitions; (ii) $0.9>A R I \geq 0.8$ suggests good agreement; (iii) $0.8>A R I \geq 0.65$ can be viewed as moderate agreement; and (iv) $A R I \leq 0.65$ indicates poor agreement between partitions. According to those rules we will say that blockmodeling is stable in terms of partitions if the mean value of the Adjusted Rand Index ( $m A R I$ ) is above 0.8 .

The second index is the proportion of incorrect block types $(\operatorname{Err} B)$ which measures the agreement between block types and their locations in the real and 'simulated' blockmodels. It is calculated as the number of block disagreements divided by the number of blocks in the blockmodel ${ }^{7}$. If the two blockmodels agree perfectly then $\operatorname{Err} B=0$ and when the two image matrices disagree regarding the locations of block types then $\operatorname{Err} B>0$. For the Adjusted Rand Index we said that the blockmodel is acceptable if the $m A R I$ is above 0.8 which indicates that we have $20 \%$ of the highest possible $A R I$ values in the acceptance region. To make both indices of blockmodeling stability comparable in some way we decided that again $20 \%$ of the best possible $\operatorname{Err} B$ values will indicate acceptable blockmodel. Therefore, if the mean value of proportion of incorrect block types $(m E \operatorname{Er} B)$ is below 0.2 we say that blockmodeling is stable in terms of blockmodel structure and its composition.

\footnotetext{
${ }^{6}$ For each real network and selection of number of fixed choices ( $n$ Fixed) the blockmodeling procedure was repeated 100 times.

${ }^{7}$ It is assumed that the two blockmodels have the same number of positions and hence blocks. Otherwise, the blockmodel partition is regarded as a very poor blockmodel and it is not considered further.
} 


\subsection{Two real networks}

\subsubsection{Boy-girl liking network}

The first real network has 11 actors (left panel of Figure 1) and presents a liking relation between boys and girls in a classroom (Doreian et al., 2005: 237). These data were collected by a student in a social network analysis class at the University of Pittsburgh. The data come from a pre-school where the measured relation was 'plays with' and was constructed by observing playing activity among children. The relation was later interpreted as 'liking'. In the network data collection process the free choice design was used without limitation of the number of actors. There are two groups, based on gender, which were revealed also by blockmodeling procedure based on structural equivalence (right panel of Figure 1). The image matrix has two complete blocks on the diagonal and null blocks out of diagonal. There are 12 inconsistencies which are all null ties within the two diagonal blocks.

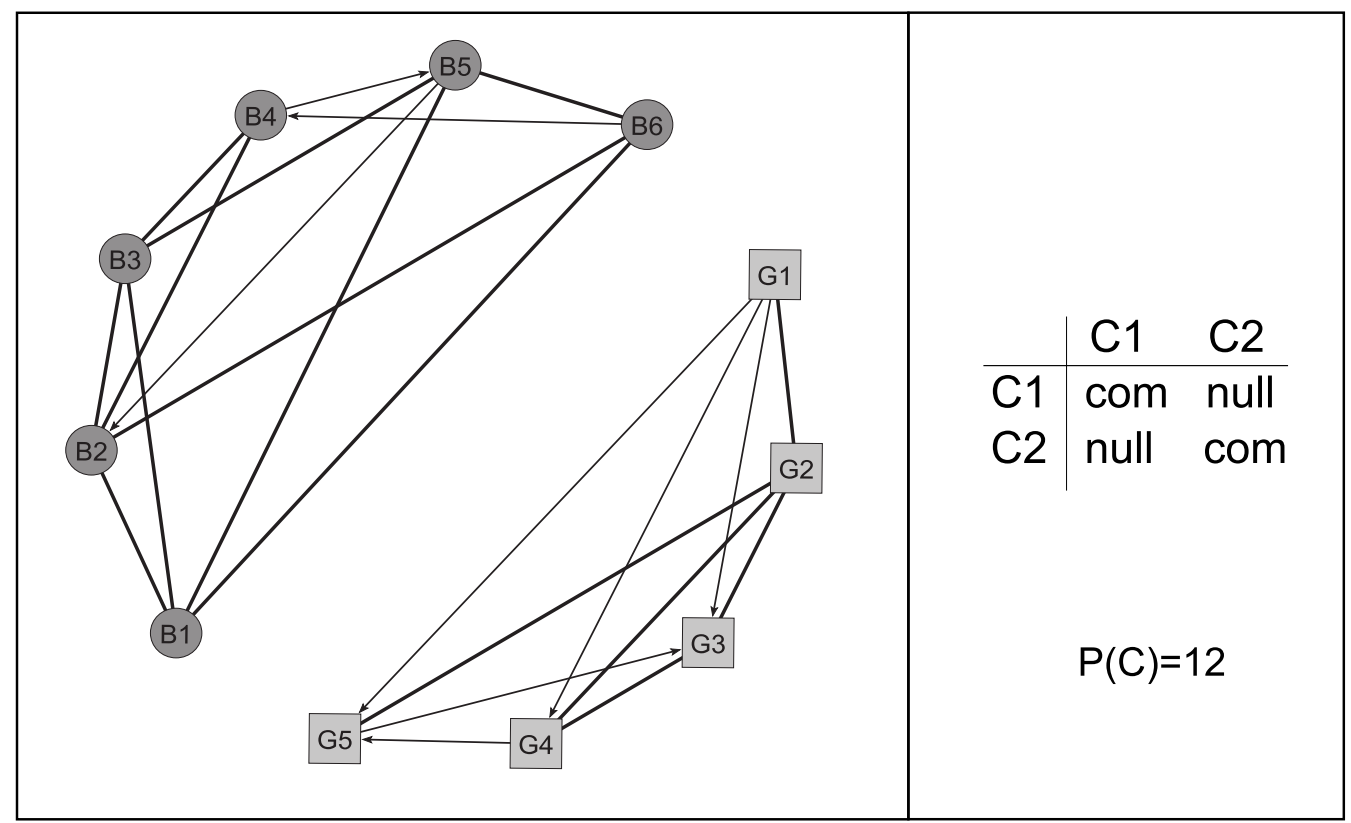

Figure 1: Boy-girl liking network s (left) with two partitions based on structural equivalence and image matrix (right).

\subsubsection{Student note borrowing network}

Actors in a note borrowing network are undergraduate students attending lectures. The network data were collected with free choice design questionnaire by Hlebec (Hlebec and Ferligoj, 2002) and used by Batagelj et al. (2004: 460). The students were asked: "from whom would you borrow learning materials?" The network is presented as a graph in Figure 2 (left panel). Boys are represented by squares and the girls by circles, while the shading of the vertices indicates position memberships of three clusters (labeled as $\mathrm{C} 1$, C2, and C3) from obtained blockmodeling using structural equivalence. Black indicates membership in cluster $\mathrm{C} 1$, white shows membership in $\mathrm{C} 2$ and gray indicates membership 
in C3. The fitted blockmodel with 28 inconsistencies has three complete blocks (right panel of Figure 2).

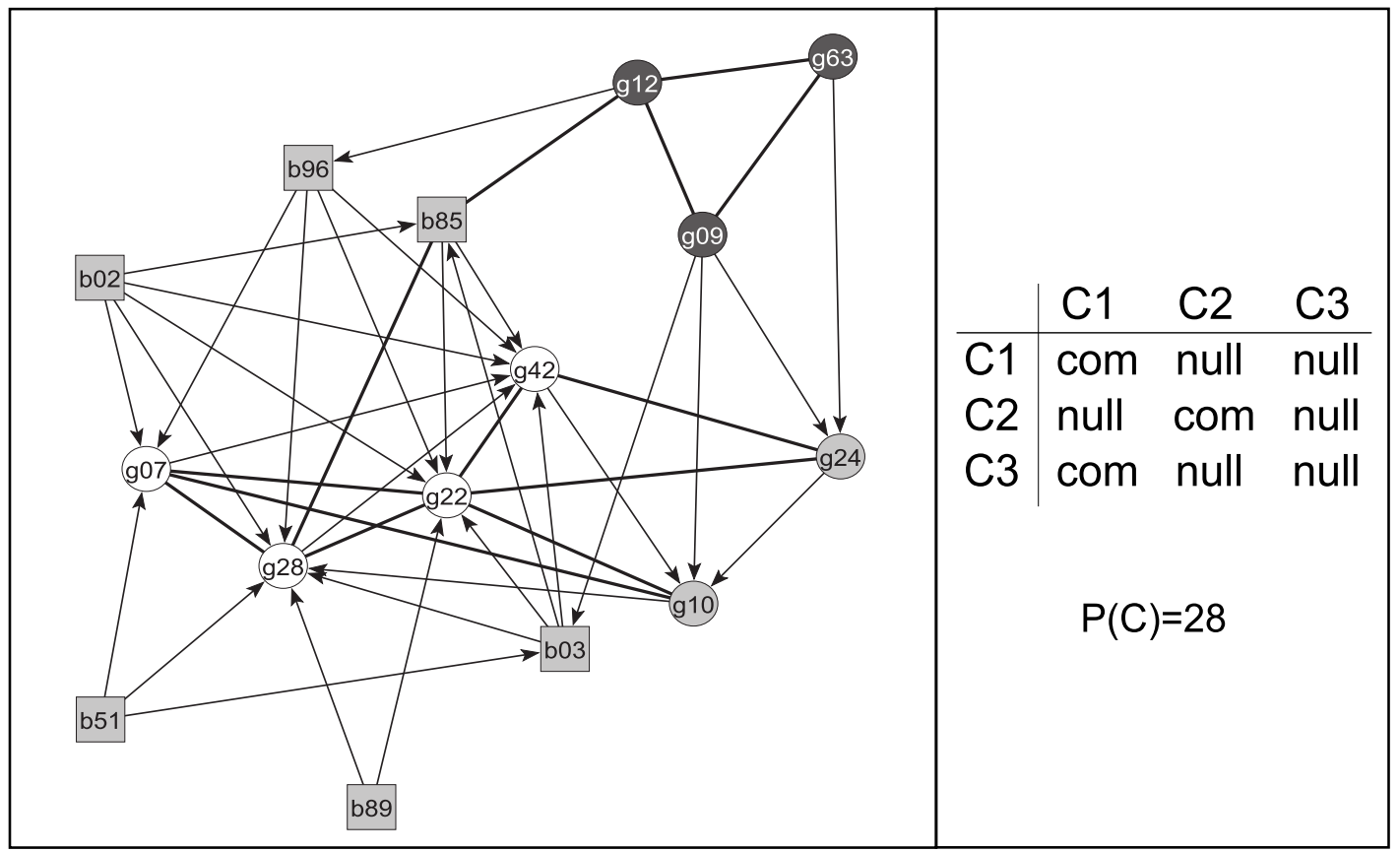

Figure 2: Student note borrowing network (left) with three partitions based on structural equivalence and image matrix (right).

\section{Results of simulation study}

\subsection{Boy-girl liking ties network}

In the simulation study, for each selected number of considered fixed number of choices, 100 networks were generated. The simulated network was generated with random addition or deletion of ties, so that the condition concerning the limitation of ties was satisfied. Blockmodels of the simulated networks were established based on structural equivalence and compared with the blockmodel of real network using both indices of blockmodeling stability (the Adjusted Rand Index and the proportion of incorrect blocks).

Actors in the boy-girl liking network selected from two to four friends and the average number of choices made (average outdegree) is 3.45 (with standard deviation $0.82, Q_{1}=$ $3, M e=4, Q_{3}=4$ ). Two actors (G3 and G5) made only two choices, three nominations were made by actors B4 and G4, other seven actors selected four other members of the network. Based on those results we may suspect that restriction of number of choices to 3 or 4 actors will not radically change the blockmodel structure of the simulated network, because a small proportion of ties would be changed in that case.

The fixed choice design was simulated with a range of the restriction for nominations from one to seven actors. Agreement for the partitions of actors was measured with the Adjusted Rand Index and is presented with boxplots in Figure 3(a), while mean values of $A R I$ are presented with black dots. 


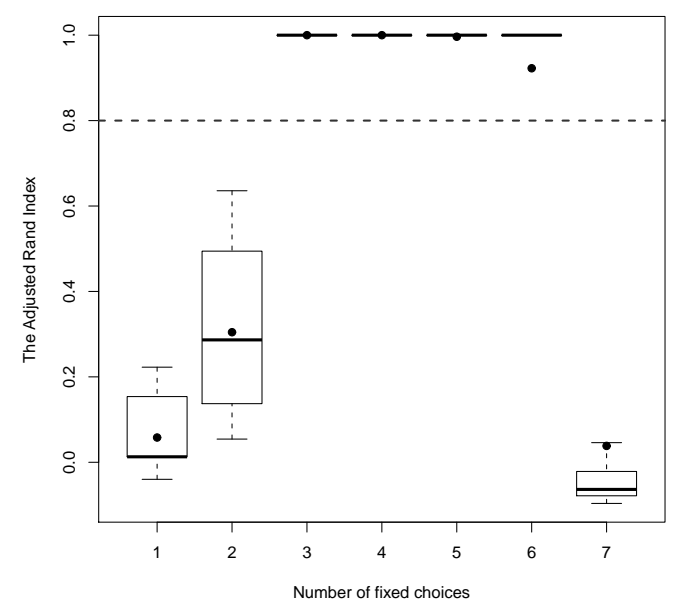

(a) Mean of the Adjusted Rand Index, $m A R I$

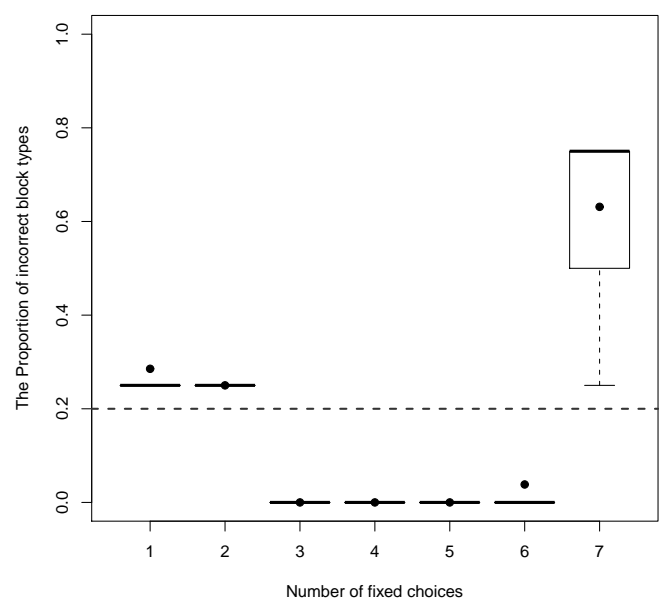

(b) Mean of Incorrect block types, $m \operatorname{Err} B$

Figure 3: Results of the simulation study with the boy-girl liking ties network for simulated fixed choice designs.

As expected, the blockmodeling is stable in terms of partitions when the number of choices is set to three or four nominations because these restrictions are more realistic according to the whole real network. In fact, the agreement across the partitions is perfect: $m A R I=1$ for each of the 100 measured partitions and the real partition of the whole blockmodeling presented in Figure 1. The agreement between partitions is also almost perfect if the number of fixed choices is equal to five. Acceptable agreement across partitions $(m A R I>0.8)$ is obtained with random simulation of six choices, while the increase of number of fixed choices to seven nominations leads to complete disagreement between partitions with $m A R I$ values around zero. If the number of fixed choices is low (one or two nominations), then the ties are deleted from the real boy-girl liking network in order to satisfy the restrictions. Therefore, the simulated structure is poorer than the real structure of tie patterns and the agreement between partitions is unacceptable.

Figure 3(b) presents the stability of blockmodeling in terms of a correctly identified block structure. As written above, the agreement between two blockmodels (or image matrices) is acceptable if the mean values of proportion of incorrectly identified block types $(m E r r B)$ do not exceed 0.2. Perfect agreement between image matrices is obtained if fixed choice nominations are restricted to three, four or five choices $(m E \operatorname{Er} B=0)$. Acceptable agreement between block structures is also obtained with six choices. On the other hand, a small number (one or two) or high number (seven nominations) of fixed choices leads to unacceptable agreement between blockmodels with $m \operatorname{Err} B$ higher than 0.2. If the number of choices is restricted to one or two nominations, the mean proportion of incorrectly identified block types is around 0.25: one block (out of four) in a blockmodel is incorrectly classified.

As noted above, if the number of choices is restricted to one or two nominations, then some ties are deleted from the whole boy-girl liking ties network. Actors in the network nominate from two to four friends, therefore the restriction of choices to three 
nominations will lead to both deletion and addition of ties. The ties are randomly added to the network, if the restriction of choices is set to four or more nominations. In these cases, the observed real structure is a subset of simulated network and actors have to add non-existing ties to meet the threshold criterion. Therefore, we focus on the relationship between the percentage of ties that changed and the different restriction rules according to both indices.

Figure 4(b) presents mean values of $A R I$ plotted against the percentage of changed ties and allows us to compare both error mechanisms, randomly introduced measurement errors $^{8}$ and limitation of the number of choices. Gray points indicate mean values of $A R I$ according to the percentage of randomly changed ties in the boy-girl liking network. If the simulated ties were randomly deleted to satisfy the limitation criteria the results are presented with white boxplots, if ties were only randomly added the results are plotted in black, and the gray color indicates results obtained with both randomly added and randomly deleted to meet the specified criterion about the number of nominations.

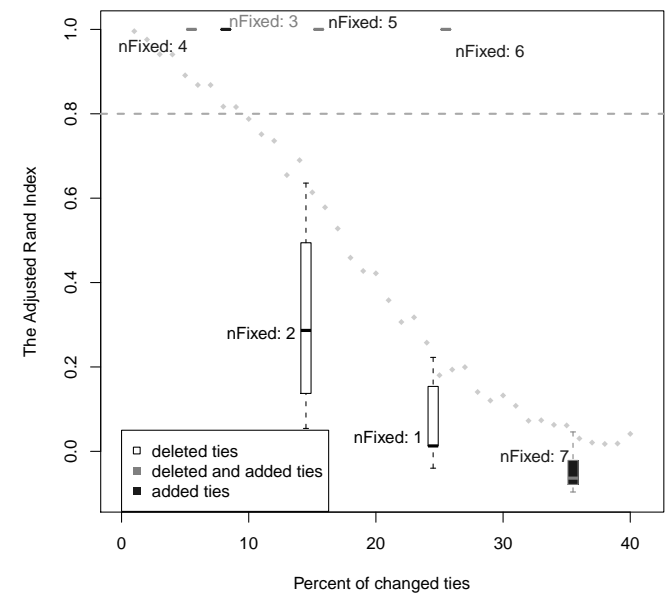

(a) Mean of the Adjusted Rand Index, $m A R I$

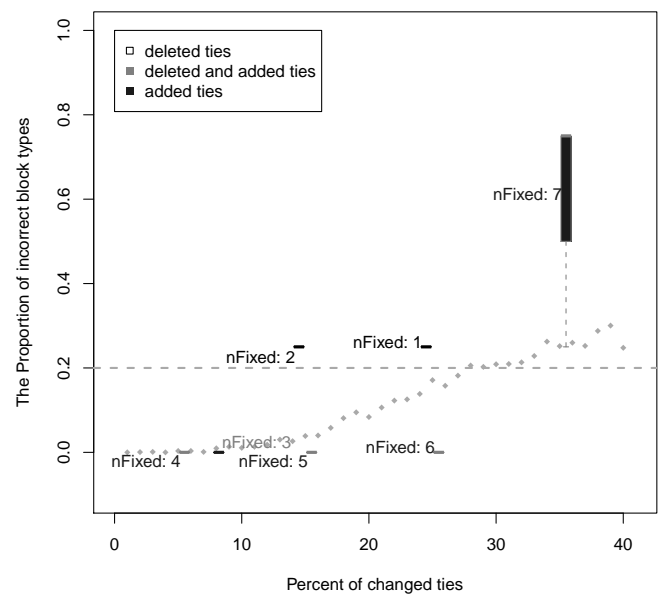

(b) Mean of Incorrect block types, $m \operatorname{Err} B$

Figure 4: The percentage of changed ties in the simulation study for the boy-girl liking network for fixed choice designs.

The smallest percentage of changed ties $(6 \%)$ is obtained if the number of nominations was restricted to four choices. There is a perfect agreement between simulated and real partition if the ties were only added to the measured networks. With five required nominations $16 \%$ of ties were changed (more precisely, some ties were added) in the simulated network and the agreement between partitions is still perfect. The result is similar if $16 \%$ of ties were randomly changed (one or a tie, is replaced by zero and and vice versa). More than quarter (26\%) of ties were added to the network if the number of fixed choices was equal to six, but the agreement between partitions was still acceptable with mean value of $A R I$ values around 0.9. Compared to the same percentage of randomly changed ties, the results for fixed choice mechanism are better. Interesting results were obtained if we compared restrictions to two and five nominations, where $14 \%$ and $16 \%$ of

\footnotetext{
${ }^{8}$ The extensive results on randomly introduced measurement errors can be found in Žnidaršič (2012).
} 
ties were changed, respectively. In the first case ( 2 fixed choices) ties were deleted from the real network and the result for $m A R I$ is overwhelming $(m A R I \approx 0.3)$. In the second case (five fixed choices) higher percent of ties was changed, but ties were added to the network. The agreement between both partitions in this case is acceptable. Therefore, we conclude that addition of ties in a fixed choice design is less destructive than deleting ties. In other words, if the study design requires fixed choice, the restriction of the nominations should not be set too low.

The results for the percentage of incorrectly identified block structure plotted against the percentage of changed ties are presented in Figure 4(b). As for $m A R I$, the deletion of ties when the number of choices is limited to one or two nominations destroys the blockmodel structure, and values of $m \operatorname{Err} B$ are higher compared to the case where the same percentage of ties were randomly added (restriction to five and six actors) or added and deleted (three actors are requested to nominate).

\subsection{Student note borrowing network}

The student note borrowing network has 15 actors. The blockmodel based on structural equivalence into three clusters is in Figure 2. The average outdegree (the average number of nominations) is 3.73 (with a standard deviation of $0.88, Q_{1}=3, M e=Q_{3}=4$ ). One actor nominated only one member, five actors nominated three other members, six actors made four nominations and three actors nominated five other members of the network.

Limitation of the number of choices was simulated within the range from one to nine actors. Figure 5(a) presents the results for stability of blockmodeling based on structural equivalence in terms of partitions into three clusters. The mean values of $A R I$ are acceptable for four to five nominations because $m A R I>0.8$. For fixed choice equal to six or higher, the $m A R I$ decreased to zero indicating unacceptable agreement between partitions. Mean values of $A R I$ are below 0.8 also for limitations from one to three fixed choices.

Figure 5(b) presents the stability of blockmodels for the note borrowing network in terms of correctly identified block types $(\operatorname{Err} B)$. These results suggest that the impacts of fixed choice designs are less consequential for getting the blockmodel right than for position membership. Mean values of $\operatorname{Err} B$ are below 0.2 indicating that less then two incorrectly identified block types in a blockmodel, for whole range of fixed choices from two to seven. The blockmodel or image matrix of measured network compared to whole network is unacceptable if the restriction of number of nominations is set to one or higher than seven.

these results suggests that the impact of fixed choice designs are less consequential for getting the blockmodel right than for position membership.

As noted above, actors made two to five nominations. Therefore, if the number of choices is restricted to one or two nominations, then ties were deleted from the note borrowing network. If the number of choices is set to three or four, then ties are both added and deleted to satisfy the condition. Ties were randomly added to the network, if the restriction of choices is set to five or more nominated actors.

Figure 6(a) presents mean values of $A R I$ plotted against the percentage of changed ties. Gray shows mean values of $A R I$ when the random measurement errors are introduced to the network. The smallest percentage of changed ties $(5 \%)$ is obtained if the 


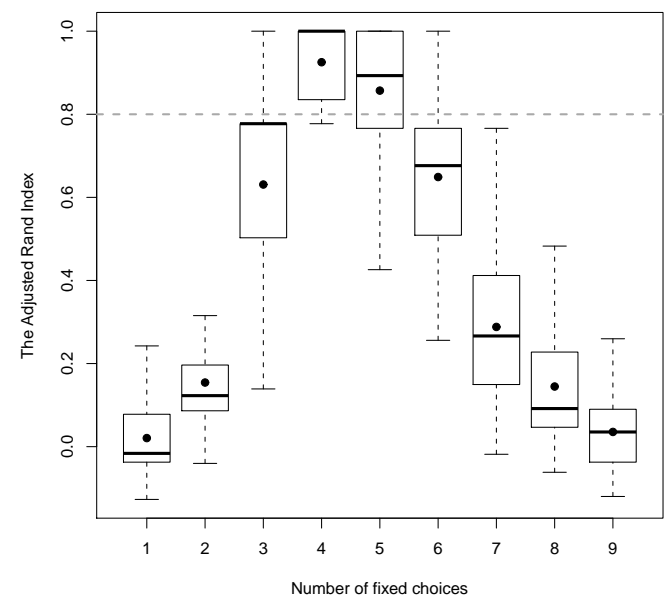

(a) Mean of the Adjusted Rand Index, $m A R I$

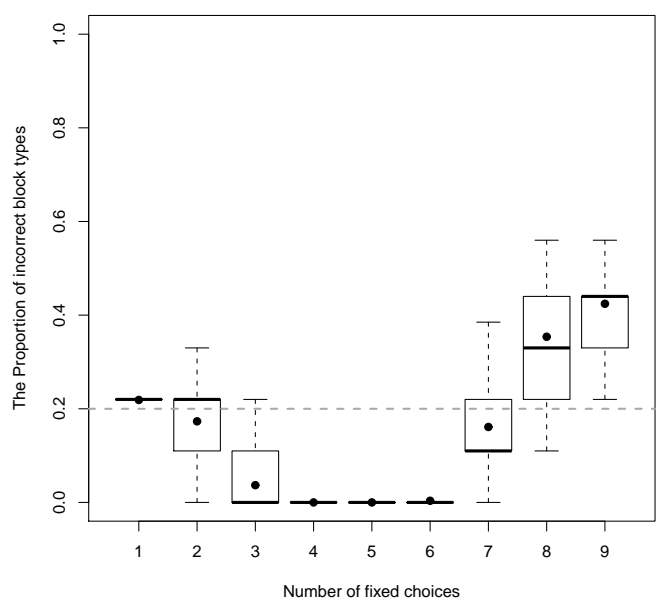

(b) Mean of Incorrect block types, $m \operatorname{Err} B$

Figure 5: Results of the simulation study for the note borrowing network for fixed choice designs.

number of nominations is restricted to four choices and the agreement between partitions is acceptable. If the choices are limited to three actors, the majority of changed ties were deleted tie and only one tie was added, because one actor has just two nominations. In this case, $6 \%$ of ties were changed and the mean value of $A R I$ indicates unacceptable agreement between partitions. When the number of choices was limited to five actors, ties were only added to the network and the agreement between partitions according to $m A R I$ is acceptable. In this case, we changed $9 \%$ to meet the limitation criteria and the $m A R I$ values are higher than in case of three choices restriction. As for the boy-girl liking network, we conclude that adding ties is less destructive than deleting ties.

A less severe effect of adding than deleting ties in a fixed choice design can also be seen in stability of blockmodels according to their block structure (Figure 6(b)). Mean values of $\operatorname{Err} B$ are below 0.2 for restricted nominations from three to seven actors, where ties were both added and deleted or just added to the real network. When ties were only deleted to meet the limitation criteria, the $m \operatorname{Err} B$ values are noticeably higher (around 0.2 ) than comparable fixed choice cases (according to the number of changed ties) where ties were added.

\subsection{Conclusions}

Given the foregoing results, we conclude that limiting the number of choices may destroy the blockmodel structure if the restriction is unrealistic or too far from the true number of ties. Newman (2010) emphasized that "limits are often imposed purely for practical purposes, to reduce the work the experimenter must do". We emphasize that this is not an appropriate reason for adopting a fixed choice questionnaire format because it has high ability to destroy the underlying true structure and estimates of network statistics (Holland and Leinhardt, 1973; Kossinets, 2006). Therefore, several authors warn against its use. 


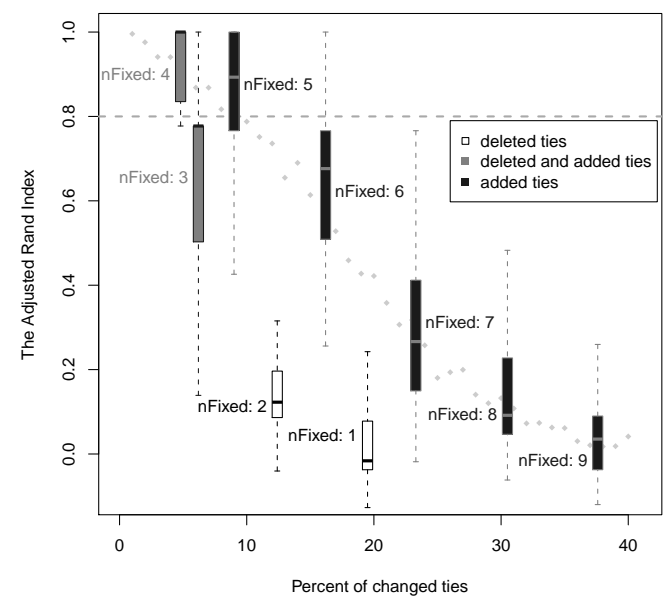

(a) Mean of the Adjusted Rand Index, $m A R I$

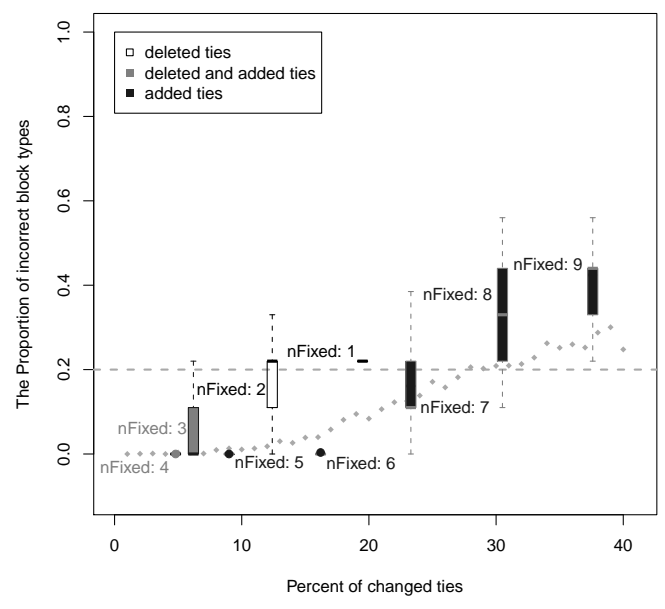

(b) Mean of Incorrect block types, $m \operatorname{Err} B$

Figure 6: Percentage of changed ties in the simulation study for the note borrowing network for fixed choice designs.

Also from a blockmodeling point of view, the questionnaire format in social network studies should not enforce a fixed number of choices. If there is a reasonable argument for using a fixed choice design, limitations should not be set too low. For example, to establish a blockmodel based on structurual equivalence it is better that the questionnaire format forces the respondents to nominate more others (than is the real number) than to restrict the number of nominations to low values where actors are not able to nominate all of the other with whom they are linked. On the other hand, the limitation of number of actors should not be set to high. To obtain acceptable blockmodel structure and the partition of actors the limitation of number of actors should be as realistic as possible and should not exceed approximately $40 \%$ of actors in a network (according to our examples).

In combination with the free choice design questionnaires we also recommended using a roster because it reduces the number of of forgotten ties and therefore provides richer structure of ties among actors (Hlebec, 1993; Hlebec and Ferligoj, 2001; Brewer, 2000; Brewer and Webster, 2000). The use of a roster also simplifies the reporting task for actors by reminding them of eligible network members (Marsden, 2011).

The main limitation of this study is the fact that simulations was performed only with two small network data sets. Therefore, the extensive simulations with broader set of empirical networks and also with simulated networks with desired blockmodel structure should be performed in the further studies. Another limitation is that in simulations of fixed choice design ties were randomly added or deleted (or both) to satisfy the selected limitation criteria. In further research this procedure should be extended to other techniques where for example popularity of actors should be taken into account. More precisely, the probability of the addition or the deletion of ties for an actor should be proportional to the indegree of that actor. 


\section{References}

[1] Batagelj, V., Doreian, P. and Ferligoj, A. (1992a): An optimizational approach to regular equivalence. Social Networks, 14, 121 - 135.

[2] Batagelj, V., Ferligoj, A. and Doreian, P. (1992b): Direct and indirect methods for structural equivalence. Social Networks, 14, 63-90.

[3] Batagelj, V. and Mrvar, A. (2012): Pajek 3.05 - program for Large Network Analysis. Available at: http://pajek.imfm.si/doku.php?id=download.

[4] Batagelj, V., Mrvar, A., Ferligoj, A., and Doreian, P. (2004): Generalized Blockmodeling with Pajek. Metodološki Zvezki, 1, 455-467.

[5] Bell, D.C., Belli-McQueen, B., and Haider, A. (2007): Partner naming and forgetting: Recall of network members. Social Networks, 29, 279-299.

[6] Borgatti, S. P. and Everett, M.G. (2006): A graph-theoretic perspective on centrality. Social Networks, 28, 466-484.

[7] Brewer, D.D. (2000): Forgetting in the recall-based elicitation of personal and social networks. Social Networks, 22, 29-43.

[8] Brewer, D. D. and Webster, C.M. (2000): Forgetting of friends and its effects on measuring friendship networks. Social Networks, 21, 361-373.

[9] Costenbader, E. and Valente T.W. (2003): The stability of centrality measures when networks are sampled. Social Networks, 25, 283-307.

[10] Doreian, P., Batagelj, V., and Ferligoj, A. (2005): Generalized Blockmodeling. Cambridge University Press, New York, NY.

[11] Doreian, P. and Woodard, K.L. (1994): Defining and locating cores and boundaries of social networks. Social Networks, 16, 267-293.

[12] Feld, Scott L. (1991): Why your friends have more friends than you do. The American Journal of Sociology, 96, 464-1477.

[13] Feld, S.L. and Carter, W.C. (2002): Detecting measurement bias in respondent reports of personal networks. Social Networks, 24, 365-383.

[14] Ferligoj, A. and Hlebec, V. (1999): Evaluation of social network measurement instruments. Social Networks, 21, 111-130.

[15] Hlebec, V. (1993): Recall versus recognition: Comparison of the two alternative procedures for collecting social network data. In Ferligoj, A. and Kramberger, A. (Eds.): Metodolški Zvezki, Developments in statistics and methodology, 9, 121-128. FDV, Ljubljana.

[16] Hlebec, V. and Ferligoj, A. (2002): Reliability of social network measurement instruments. Field method, 14, 288-306. 
[17] Hlebec, V. and Ferligoj, A. (2001): Respondent mood and the instability of survey network measurements. Social Networks, 23, 125-139.

[18] Hlebec, V. and Kogovšek (2006): Merjenje socialnih omrežij. Študentska založba, Ljubljana. In Slovene.

[19] Holland, P.W. and Leinhardt, S. (1973): The structural implications of measurement error in sociometry. The Journal of Mathematical Sociology, 3, 85-111.

[20] Hubert, L. and Arabie, P. (1985): Comparing partitions. Journal of Classification, 2, 193-218.

[21] Huisman, M. (2009): Effects of missing data in social networks. Journal of Social Structure, 10. Available at: http://www.cmu.edu/joss/content/articles/volume10/huisman.pdf.

[22] Huisman, M. and Steglich, C. (2008): Treatment of non-response in longitudinal network studies. Social networks, 30, 297-308.

[23] Kogovšek, T., Mrzel, M., and Hlebec, V. (2010): "Please name the first two people you would ask for help": The effect of limitation of the number of alters on network composition. Metodološki Zvezki, 7, 95-106.

[24] Kogovšek, T. and Hlebec, V. (2005): Effects of limitation of number of alters and time frame in the Burt name generator. Metodološki zvezki, 2, 59-71.

[25] Knoke, D. and Yang, S. (2008): Social Networks Analysis. Sage Publications,Los Angeles. 2nd edition.

[26] Kossinets, G. (2006): Effects of missing data in social networks. Social networks, 28, 247-268.

[27] Laumann, E.O., Marsden, P.V., and Prensky, D. (1983): The boundary specification problem in network analysis. In Burt, R. S. and Minor, M. J. (Eds.): Applied Network Analysis, 18-34. London: Sage Publications.

[28] Lorrain, F. and White, H.C. (1971): Structural equivalence of individuals in social networks. Journal of Mathematical Sociology, 1, 49-80.

[29] Lozar Manfreda, K., Vehovar, V., and Hlebec, V. (2004): Collecting egocentred network data via the web. Metodološki Zvezki, 1, 295-321.

[30] Marsden, P.V. (2005): Recent developments in network measurement. In Carrington P.J., Scott J., and Wasserman S. (Eds.): Models and Methods in Social Network Analysis, 8-30. New York: Cambridge University Press.

[31] Marsden, P.V. (2011): Survey methods for network data. In Scott J. and Carrington P.J. (Eds.): The SAGE Handbook of Social Network Analysis, 370-388. Thousand Oaks: Cambridge Sage Publications. 
[32] Mrvar, A. and Batagelj, V. (2012): Pajek and Pajek - XLL, Programs for Analysis and Visualization of Very Large Networks, Reference Manual, List of Commands with Short Explanation, version 3.05.

Available at: http://pajek.imfm.si/lib/exe/fetch.php?media=dl:pajekman305.pdf.

[33] Newman, M.E.J. (2003): Ego-centered networks and the ripple effect. Social Networks, 25, 83-95.

[34] Newman, M.E.J. (2010): Networks. An Introduction. New York: Oxford University Press Inc.

[35] Rumsey, D.J. (1993): Nonresponse models for social network stochastic processes. $\mathrm{Ph}$.D. thesis, The Ohio State University.

[36] Steinley, D. (2004): Properties of the Hubert-Arabie Adjusted Rand index. Psychological Methods, 9, 386-396.

[37] Stork, D. and Richards, W.D. (1992): Nonrespondents in communication network studies: problems and possibilities. Group and Organization Management, 17, 193209.

[38] Vehovar, V., Lozar Manfreda, K., Koren, G., and Hlebec, V. (2008): Measuring egocentered social networks on the web: Questionnaire design issues. Social Networks, 30, 213-222.

[39] Wasserman, S. and Faust, K. (1994): Social Network Analysis: Methods and Applications. New York: Cambridge University Press.

[40] Žiberna, A. (2008): Blockmodeling 0.1.7: An R package for Generalized and classical blockmodeling of valued networks. Available at: http://www2.arnes.si/ aziber4/

[41] Žnidaršič, A. (2012): Stability of blockmodeling. Ph.D thesis of University of Ljubljana, Ljubljana.

[42] Žnidaršič, A., Doreian, P., and Feligoj, A. (2012a): Tie non-response in social networks, treatments of tie non-response, and blockmodeling outcomes. Social Networks, doi: http://dx.doi.org/10.1016/j.socnet.2012.02.002

[43] Žnidaršič, A., Doreian, P., and Feligoj, A. (2012b): Absent ties in social networks, their treatments, and blockmodeling outcomes. Metodološki Zvezki, 9, 119-138.

[44] Žnidaršič, A., Feligoj, A., and Doreian, P. (2012c): On the stability of blockmodels based on structural and regular equivalence given measurement errors. In Analysis and modeling of complex data in behavioural and social sciences: book of abstracts. JCS - CLADAG 12 / Joint meeting of the Japanese Classification Society and the Italian Classification and Data Analysis Group, 96. Anacapri, Italy. 